\title{
Medicine on the Internet
}

\author{
M. MichaEL SHABOT, MD
}

$\mathrm{T}$ he Internet, to many, is a mystery. People often have 3 questions about it: Who runs it? How big is it? Who's paying for it? In this paper, I provide some basic information about the Internet and focus on medical uses of this technology.

\section{HISTORY OF THE INTERNET}

The true birth of the Internet is dated from October 4, 1957, when Sputnik was launched. This $183-1 b$ satellite contained only a small radio transmitter but caused a palpable fear across the USA. In this Cold War period, when Americans heard the beep from the radio, they imagined a circling hydrogen bomb able to be deorbited in a matter of minutes or hours. To deal with the perceived science and technology gap, the US government founded the Advanced Research Projects Agency (ARPA). Decades later, we know that there was not much of a technological gap. Instead, there was simply a difference in the initial postwar accomplishments of the German rocket scientists who went to Russia vs those who went to the USA.

In addition to that paranoia, in 1961, a radical militia group blew up 4 microwave towers in Utah and cut off communications to the western USA for a period of time. That event highlighted the vulnerability of our existing point-to-point communications network. In response, in the early 1960s the Rand Corporation proposed a new decentralized network for defense command and control, and in 1968 the first contracts were drawn to develop the special type of message processors that would be required for the system.

The first ARPANET node was installed at the University of California at Los Angeles in September 1969. By December of that year, 4 sites were connected by the ARPANET: Stanford Research Institute, the University of California at Santa Barbara, the University of California at Los Angeles, and the University of Utah. By 1971, there were 15 nodes with 2 transcontinental links.

From the earliest stage, there were redundant links, or different ways to reach different nodes on the Net. This was an inherent feature of the fail-safe nature of ARPANET message processors. When you send a message or upload or download a file, the information is broken down into small packets. Each of those packets has an address and may take any available path to reach its destination. If the most efficient path is not available because of an outage, the message processors automatically reroute the packets and reassemble them at the destination. That's how the Internet works. It was built to be resistant to single or even multiple points of failure or attack.

In the mid-1980s, ARPANET was split into the Internet as we know it and MiliNet, a similar parallel network that our military uses for communications, command, and control. During this time, both systems were not the kind of user-friendly Internet we are familiar with, but they did allow the passage of messages between computers.

This changed in 1990, when Tim Berners-Lee at CERN, a European particle accelerator institute, invented the hypertext markup language (HTML) that enabled what he called the "World Wide Web." His purpose was to rapidly publish highenergy physics papers so that scientists around the world could access the latest research more quickly than through printed journals.

Several years later, Marc Andreeson, a graduate student at the University of Illinois who worked at the university's particle accelerator, developed Mosaic, the first graphical browser for the World Wide Web. Mosaic allowed people to click on pictures or symbols to jump from place to place on the Internet rather than entering commands. This advance was first exposed to the public in an article in the New York Times in December 1993. Andreeson went on to found Netscape. From 1994, Mosaic and then Netscape and a number of other browsers were rapidly adopted. These had a universal user interface, allowing equal access to users of IBM-compatible personal computers (PCs), Macintosh computers, Unix workstations, and other types of machines.

In 1996, major PC and Macintosh applications became Internet-enabled, so that users of the application could immediately jump to any Internet site. Within the past 2 or 3 years, the Web browser has become the standard user interface, making the underlying operating system less important than it used to be.

The rapid adoption of the Internet has been amazing. In 1995, there were nearly 5 million Internet hosts, or computers

From the Department of Surgery and Enterprise Information Services, CedarsSinai Medical Center, Los Angeles, California.

Presented as the Allan Bookatz lectureship on August 16, 2000, at Baylor University Medical Center.

Corresponding author: M. Michael Shabot, MD, Department of Surgery and Enterprise Information Services, Cedars-Sinai Medical Center, 8700 Beverly Boulevard, Suite 8215, Los Angeles, California 90048 (e-mail: michael.shabot@cshs.org). 
that provide Web pages. By 1997, there were 16 million hosts. Based on projections, we probably have 100 million hosts on the Internet in 2000, but the exact number is difficult to count. It took radio 38 years to reach 50 million users, television 13 years, and cable television 10 years, but the Internet reached that many in only 5 years.

In addition, access now reaches worldwide. In September 1991, primarily the USA and Western Europe had access to the Internet. Gradually that increased, so that now nearly every region of the world has access not only to e-mail but to full Internet services. The breakdown of the Soviet Union and the Berlin Wall closely correlated in time with the introduction of the Internet over ordinary phone lines in Russia and the Eastern Bloc.

\section{CONTROL AND COST OF THE INTERNET}

It is not surprising that Internet access helped free the USSR and the Eastern Bloc, because the Internet does not allow a government or any other group to filter out content at national boundaries. Many people would consider a fair amount of the information passing on the Internet to be objectionable for one reason or another. France, for example, is objecting to America Online (AOL) because one of its auction houses deals with German memorabilia from World War II, the sale of which is illegal in that country. The Internet was designed to avoid control by anything or anyone. If it had a single point of control, one hydrogen bomb could destroy it. The Internet was designed to be unstoppable, and essentially it is. No one runs it. If you have a problem with or complaint about content on the Internet, there is no person or office to complain to, beyond the Web site of origin.

The original US land lines-and to the extent that they are used, satellite lines - were run by the National Science Foundation until 1995. In a planned way, that backbone was shut down and the Internet was fully commercialized and privatized, at least in the USA, with major carriers-MCI, AT\&T, IBM, Sprint, Quest, and others-deploying parallel high-speed backbones. All Internet traffic travels on commercial lines in this country. In some other countries, the lines are owned by the telecommunications department of the government.

And use of the Internet is not free. The lines are expensive to install and maintain. Where's the payment? If you have AOL at home, you pay a fee to AOL. AOL in turn has to pay MCI or Sprint or Quest or AT\&T for access to the backbone lines. The high-speed lines installed at institutions are much more expensive. Individuals and institutions generally pay a flat fee and then have unlimited access for e-mail, searching, and other uses. The trend toward flat fees is being extended to cellular phone service, long distance, and other methods of communication because there is not any inherent difference between Internet traffic and voice traffic; both are digitized and sent over the same or very similar lines.

\section{USES OF THE INTERNET}

The Internet is a very powerful tool. In recent years, much effort has been put into making it entertaining. The public now considers Web surfing comparable to television, movies, and books in terms of entertainment value. An indication of this is the fact that about 3 years ago, the sale of PCs began exceeding

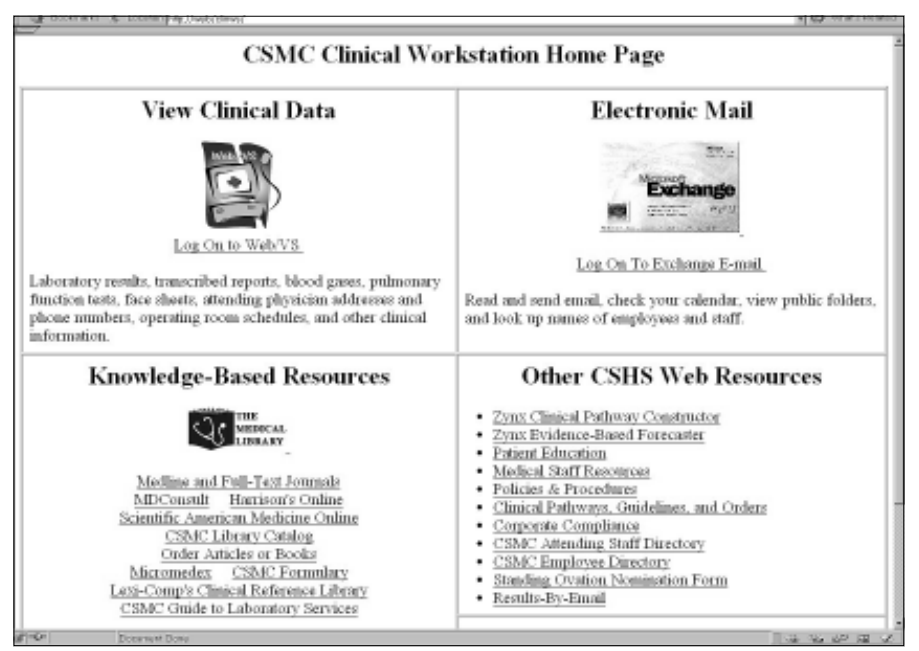

Figure 1. The home page available at Cedars-Sinai Medical Center, listing knowledge-based resource options and other information available at workstations in the hospital as well as clinical data available through log-in at any computer.

the sale of television sets in the USA. Multiuser games, virtual reality games, and real-time sound and video are now coming of age with the increased availability of higher bandwidth. It is only appropriate that health care professionals leverage all the powers of the Internet to improve patient care. What can you as a physician do on the Internet? Some uses are summarized here.

\section{E-mail}

E-mail is one of the most common uses of the Internet. Fast and free on a per-message basis, e-mail facilitates communication with colleagues and organizations, patients, and family members. E-mail messages can replace a number of other methods of communicating in a physician's practice-including managed care authorizations and referral reports and consultations. Messages can be sent to groups of people as easily as to an individual. For example, the American College of Surgeons now distributes a weekly newsletter to about 7500 fellows through email.

Use of e-mail has several advantages. Not only does it save the costs and delays associated with printing and mailing, but it eliminates phone tag and voice mail. Documents can be sent as attachments rather than being sent on a disk via US mail or Federal Express.

Related to e-mail are news groups and listservs, which users can subscribe to. Some of these deal with medical subjects. Also related are chat rooms, used primarily for entertainment. About $70 \%$ of AOL's traffic with its 22 million subscribers is in its chat rooms. People communicate with each other semianonymously and have created a new kind of real-time digital community.

\section{Finding medical information}

Several years ago, the National Library of Medicine put MedLine online for free (http://www.ncbi.nlm.nih.gov/PubMed/). Physicians can search by author's name or other key words. Abstracts are available, but full-text articles are not available at this site.

Ovid allows searches for the full text from hundreds of journals on a subscription basis. Other providers, such as MDConsult, provide similar services for a fee. At Cedars-Sinai, the follow- 


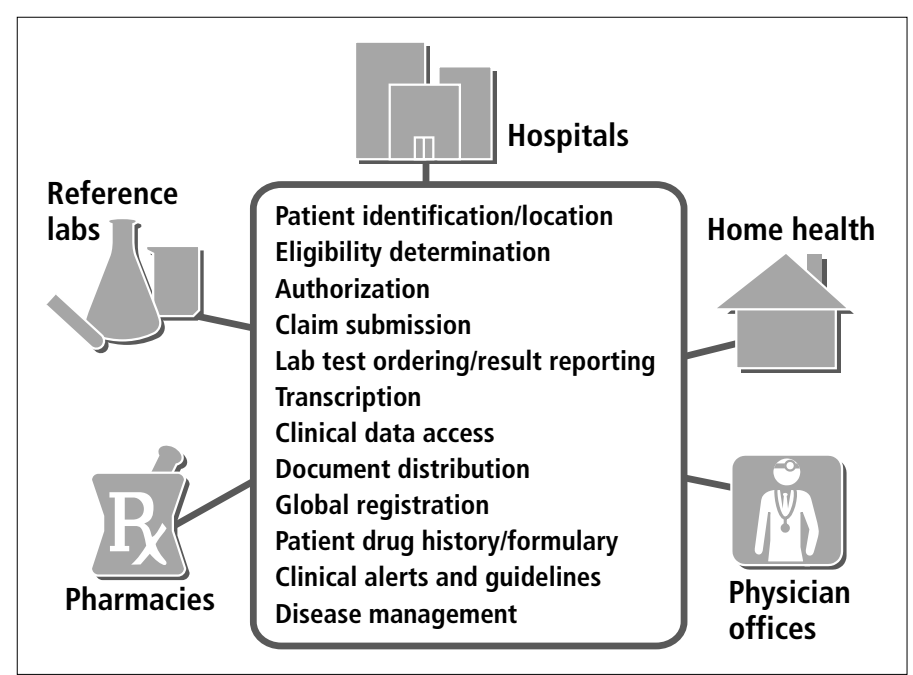

Figure 2. Proposed Internet-based clinical services.

ing databases, books, and resources are available online: Medicine and full-text journals, MDConsult, Harrison's Online, Scientific American Medicine Online, the Cedars-Sinai Medical Center library catalog, a form to order articles or books, Micromedex, the institutional formulary, Lexi-Comp's Clinical Reference Library, and the Cedars-Sinai Medical Center Guide to Laboratory Services. This information can be accessed at about 500 workstations in clinical areas (Figure 1).

The National Library of Medicine also makes available the Visible Human Project, which took an anatomically normal deceased man and woman and subjected them to high-resolution, fine-cut computed tomography, magnetic resonance imaging, and whole body sectioning, all in perfect registration for anatomic clarity (http://www.nlm.nih.gov/research/visible/visible_human. html). The data set, which is available to anyone, is about 30 gigabytes ( 15 gigabytes each for the man and the woman). The Visible Human Project also allows virtual magnetic resonance imaging endoscopy. Another useful site is Vesalius (http:// www.vesalius.com/), which has excellent anatomic drawings that can be downloaded and used in presentations.

Physicians should keep in mind the information patients can access. If patients go to a specific Web site-say, that of Baylor Health Care System (www.BaylorHealth.com) - they can look up a variety of medical conditions and also retrieve information about Baylor's cardiovascular center, transplantation center, and so on. Going to a trusted source is a good strategy, since general searches can retrieve over a million matches.

If patients prefer to do a general search for a particular problem, they can use Yahoo or any of a number of search engines. A specific search of "carotid endarterectomy" yields almost 3000 different articles. One of the problems with public access is that there is no quality control. Anyone can put medical information on a Web server, and the information retrieved may be incorrect or outdated.

\section{ADVANCED MEDICAL USES OF THE INTERNET}

The future of Internet-based medicine is to tie together many different entities: clinical services such as reference laboratories, offices, and hospitals, as well as administrative services such as pharmacies and health plans (Figures 2 and 3 ). That's the major

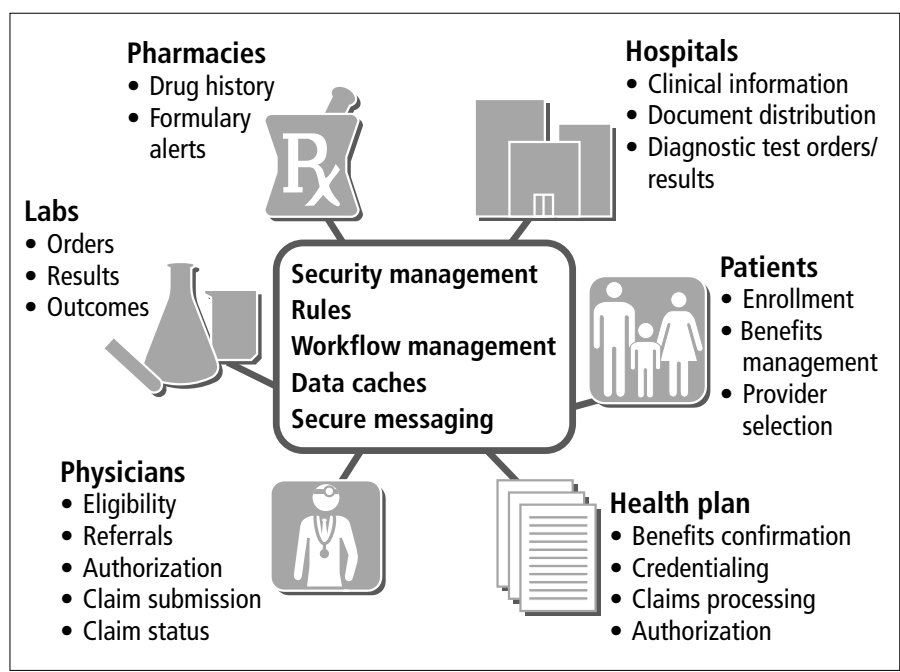

Figure 3. Proposed Internet-based administrative services.

business plan of Healtheon/WebMD, CareInsite, and other companies. Unfortunately, it is not easy to implement this convergence, as the different health care elements are not always well organized to begin with. However, some critical pieces of data have been made available on the Internet at Cedars-Sinai Medical Center, as discussed in the next section.

\section{Immediate access to patient information}

One of the great advantages of the Internet is its ease of use. If physicians know how to do 2 things on a computer, they can $\log$ in and use a Web browser. Cedars-Sinai Medical Center has taken 17 internal systems from different areas of the hospital and made them work within a single browser program, allowing physicians immediate access to patient information. This program is called "Web/VS," short for Web Viewing System.

Physicians simply connect with a modem from wherever they are. They use their browser to go to a certain Web site that Cedars-Sinai provides. The institution has a firewall and a certificate server to authenticate precisely who the user is. In addition, it provides a secure locked session with everything encrypted, which is now required by federal law for transmission of patient data.

Once physicians log in, they are taken to a page which shows their patients' names and medical record numbers. The names and numbers are hotlinked: when physicians click on them, they see the default page-recent laboratory test results for the particular patient (Figure 4). Even the test results are hotlinked, so that physicians can review the history of a white blood cell count, for example. From the default page or any other page, physicians can choose from a menu of other patient information. Menu choices include recent lab results, lab flow sheets, find any test, blood gases, images and waveforms, reports, and archived results.

Almost all of the medical records at Cedars-Sinai Medical Center are available online: operative reports, imaging reports, pathology reports, and so on (Figure 5). Physicians have access to electrocardiograms back to 1982 (Figure 6a).

The institution has its intensive care unit (ICU) and monitored bed forms online, so that for about 300 beds in the hospital, physicians can click on a patient's multilead electrocardiogram and bring up real-time waveforms and event reportings (Figure 


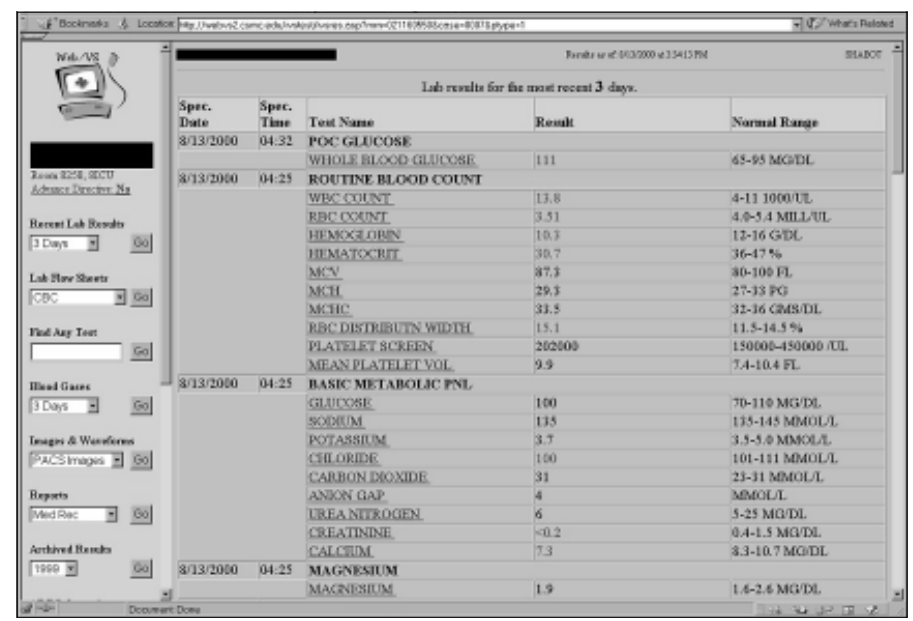

Figure 4. A screen for physicians from Cedars-Sinai's Web Viewing System showing recent laboratory values for a patient along with the menu for other data the physician can access.

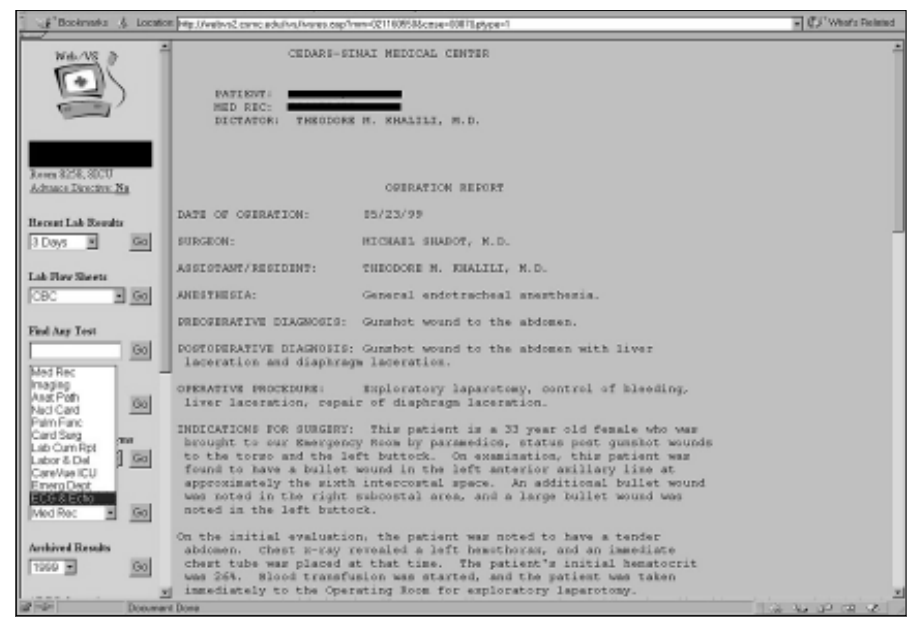

Figure 5. Sample operative report available online from Cedars-Sinai's Web Viewing System.

6b). The ICU flowsheet available for 9 different ICUs is very detailed. While this particular form is not available generally on the Internet, physicians can access an hourly flowsheet extracted from it via Web/VS at any time and from any place.

Paperless charting is also available in these 9 ICUs. Rather than writing notes for the chart by hand, physicians enter the information into the computer by constructing it from choice lists of paragraphs and phrases created in advance. At CedarsSinai Medical Center, the official record for all other areas is in paper form, but the information can be viewed electronically.

Recently, Cedars-Sinai's Picture Archiving and Communications System has become available online. The entire digital part of the imaging department can now be accessed within Web/ VS. Physicians can see a list of $\mathrm{x}$-rays, magnetic resonance images, and computed tomography images and choose one for enlargement. At that point, physicians can reverse the image, zoom in, move around the screen, and manipulate the image in other ways by clicking on easy-to-use icons (Figure 7). The resolution is not as high as it is on the dedicated workstations in the imaging department, but it is high enough so that physicians can review the films, and the imaging reports are also available in Web/ VS.
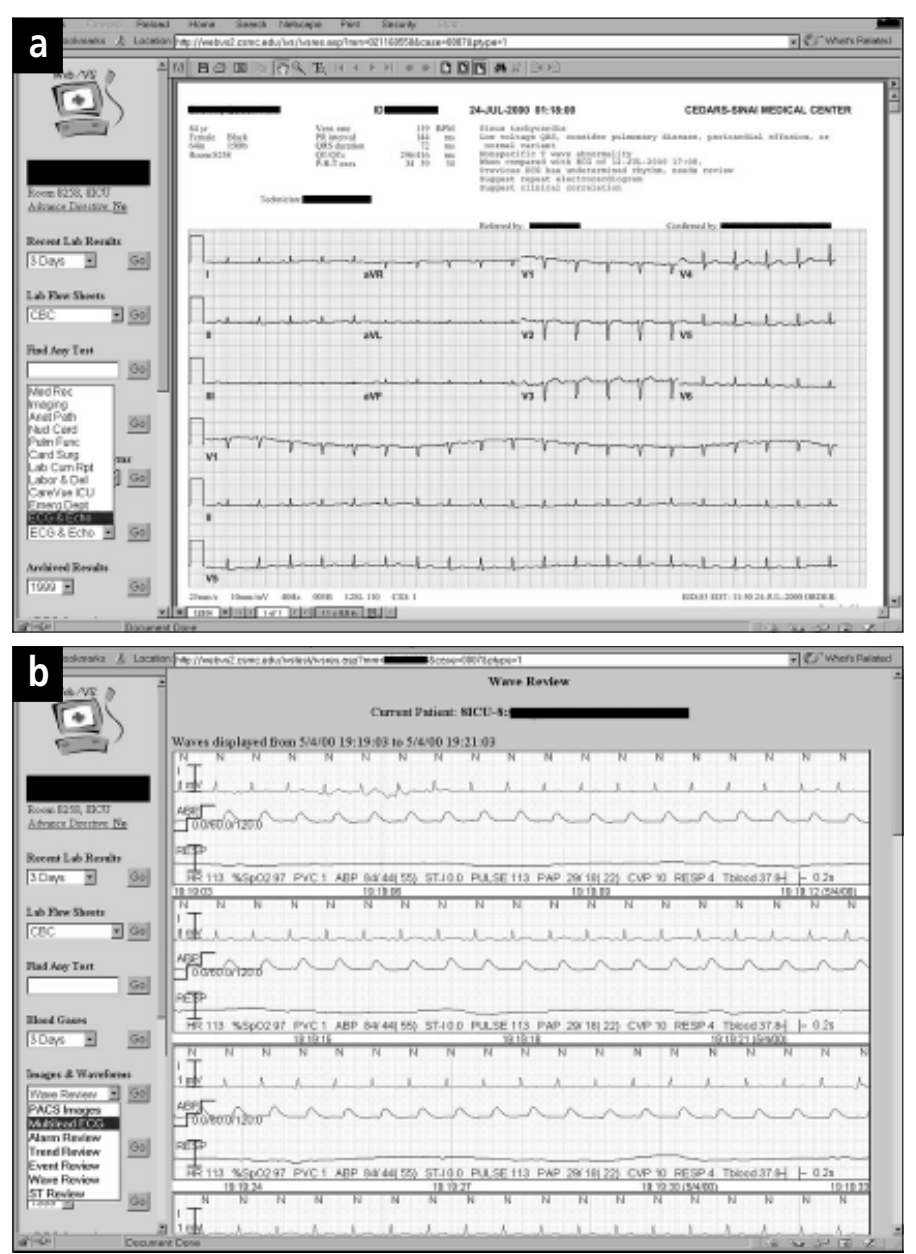

Figure 6. (a) An online electrocardiogram from Cedars-Sinai's Web Viewing System. (b) Real-time waveforms from patients in intensive care units and other monitored care areas at Cedars-Sinai. Physicians can access real-time data from workstations in the hospital and hourly extracts of intensive care unit flowsheets from any computer with Internet access, as long as they have a Cedars-Sinaiissued digital certificate to securely identify themselves.

The overall system has been up about 18 months and is now receiving $>11,000$ page hits a day. It makes phoning the laboratory and reading paper reports a thing of the past. Of course, instant access to data is not a replacement for physicians' contact with their patients; it just makes the entire staff better informed and is more convenient than older methods.

The cost of implementing the system is substantial —a single phase can cost several million dollars-but it can ultimately lead to cost savings. In addition, it allows electronic audits of pathways and guidelines, saving the time of manual extraction of chart data and increasing the speed by which results can be used to improve quality. Very few studies have been done to examine whether electronic access to data actually improves quality of care. However, Cedars-Sinai has a grant application pending to study this issue in the near future.

\section{Wireless access}

Wireless communication has led to many advances. At a more basic level, alphanumeric pagers are a move forward. More recently, send and receive pagers and modules for palmtop computers have become available, allowing the user to send responses as well as receive messages. Because Cedars-Sinai Medical Cen- 

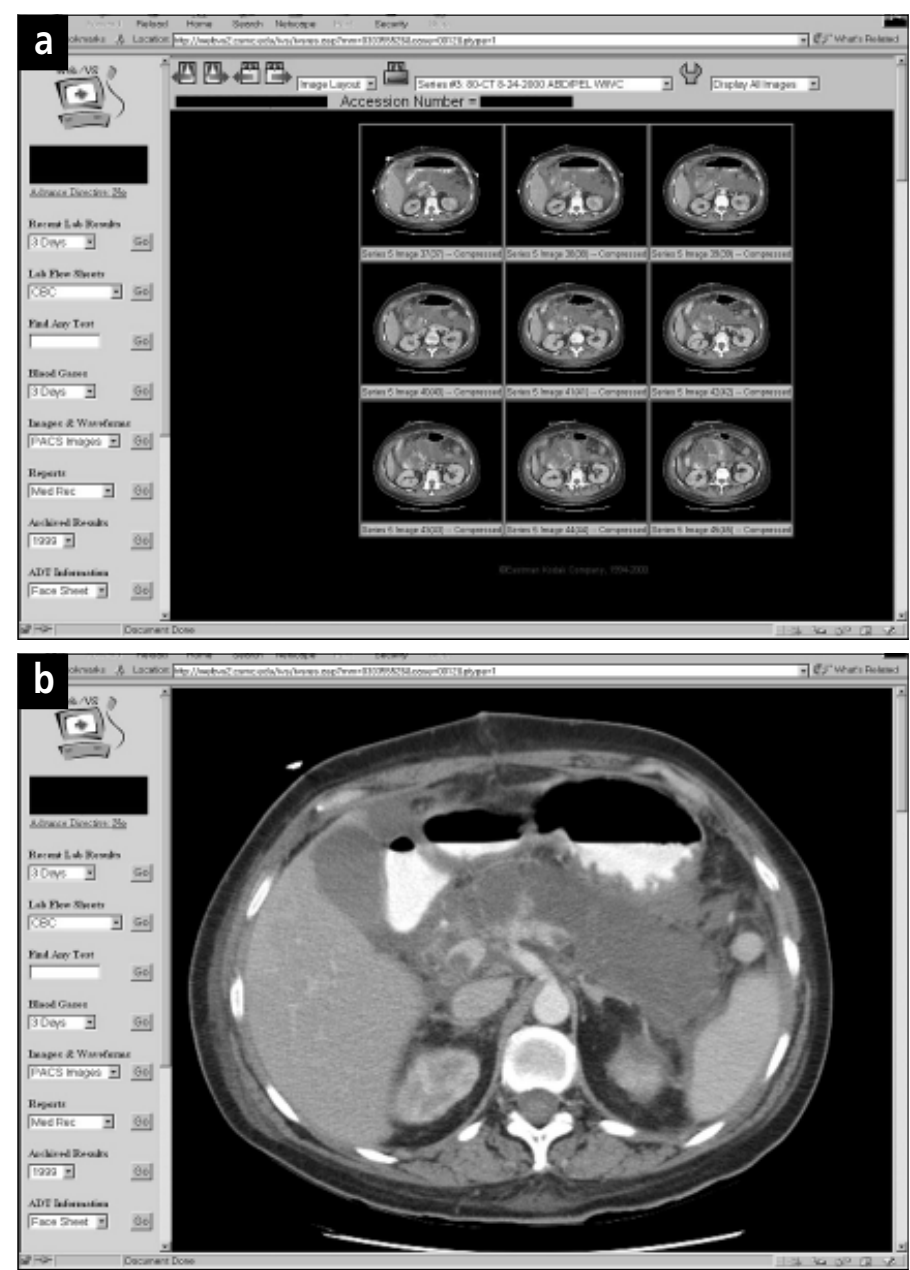

Figure 7. (a) A screen from Cedars-Sinai's Web Viewing System showing thumbnail images of an abdominal computed tomography scan. (b) Enlarged computed tomography image.

ter already has its patient data available in a secure way via the Internet, physicians can access it wirelessly as well as through computers. The Palm VII computer was selected because its wireless communication is securely encrypted. Physicians log on to $\mathrm{Palm} / \mathrm{VS}$ the same way they do at a regular computer (Figure 8a). Results are displayed in a similar fashion but are smaller due to the size of the device, as shown in an hourly flowsheet of ICU data (Figure 8b).

\section{Notification system for critical events}

Laboratory data are entered directly into the application at Cedars-Sinai Medical Center and become available on the Web immediately. Cedars-Sinai took this a step further and developed an automatic notification system for critical events. For example, laboratory data are run through computer algorithms, and if an alert condition is identified, computer-to-computer communication occurs to notify the attending physician automatically.
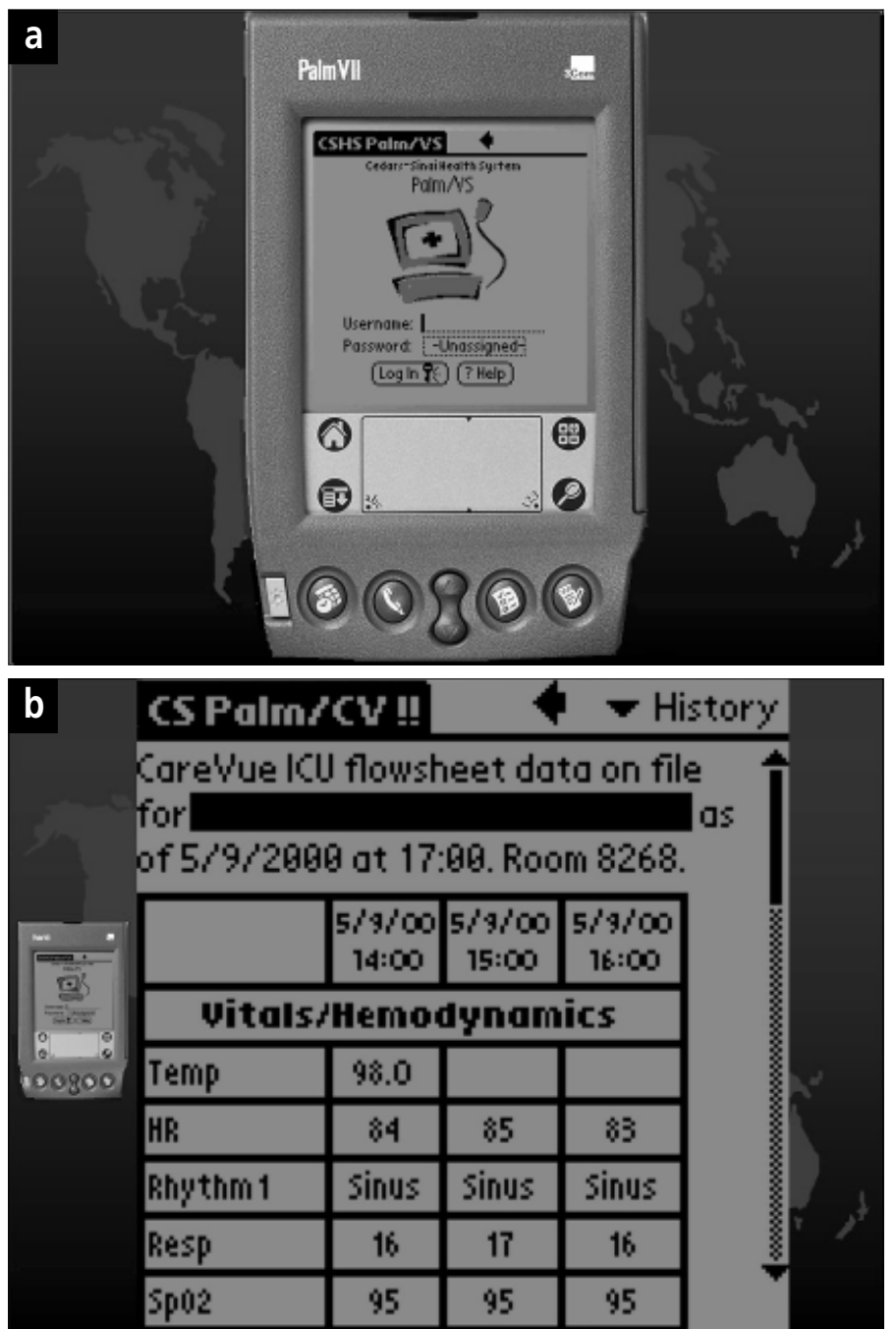

Figure 8. (a) Log-on screen for the Palm VII to wirelessly access Cedars-Sinai's Palm Viewing System (Palm/VS). (b) Wireless Palm VII access to intensive care unit flowchart information, including hourly vital signs and ventilator settings (off-screen, below).

Physicians can receive these alerts on their alphanumeric pagers. Wireless communication allows the physicians to access other patient data and send a message in response-to residents, for example—as soon as they receive the alert. This system was patented in 1995.

\section{CONCLUSION}

Universal networking, a common user interface, and Web computers are available at an extremely low cost. We are witnessing the era of "digital convergence." Everything is moving together: voice, data, wireless pagers, video on demand, cellular. Flat-rate communications are abounding, and we are seeing a tremendous transformation of traditional publishing, software, broadcast news, and other media in health care. 